\title{
Risk of dementia and Alzheimer disease increases with occupational pesticide exposure
}

O ccupational exposure to pesticides increases the risk of developing dementia and Alzheimer disease (AD) in later life, according to a longitudinal population-based cohort study published in Neurology. The results of this study provide further evidence that certain environmental factors are risk factors for these debilitating conditions.

Long-term exposure to pesticides has been hypothesized to have deleterious effects on the nervous system. Indeed, a growing body of evidence indicates that pesticide exposure might increase the risk of developing Parkinson disease. Few studies have, however, investigated an association between pesticide exposure and risk of AD. Furthermore, "these studies were relatively small and the findings have been equivocal," explains Kathleen Hayden (Duke University Medical Center, Durham, USA) lead author of the new study.

To determine whether occupational exposure to pesticides is associated with an increased risk of incident dementia and AD later in life, Hayden and colleagues conducted a longitudinal populationbased study in a well-characterized cohort of elderly individuals residing in an agricultural community. Cognitive status and information regarding pesticide exposure were obtained from the study participants at baseline, and cognitive status was subsequently monitored at three follow-up visits $\approx 3$ years, 7 years and 10 years after the start of the study.

Analysis of the results revealed that of the 3,084 individuals who fulfilled the study exclusion-inclusion criteria, 572 people reported to being exposed to pesticides during their working life. $87.8 \%$ of these individuals were men.

500 of the study participants developed incident dementia during the study, of which 344 were diagnosed with AD. Using Cox proportional hazards survival analysis, the investigators found that an increased risk of dementia was associated

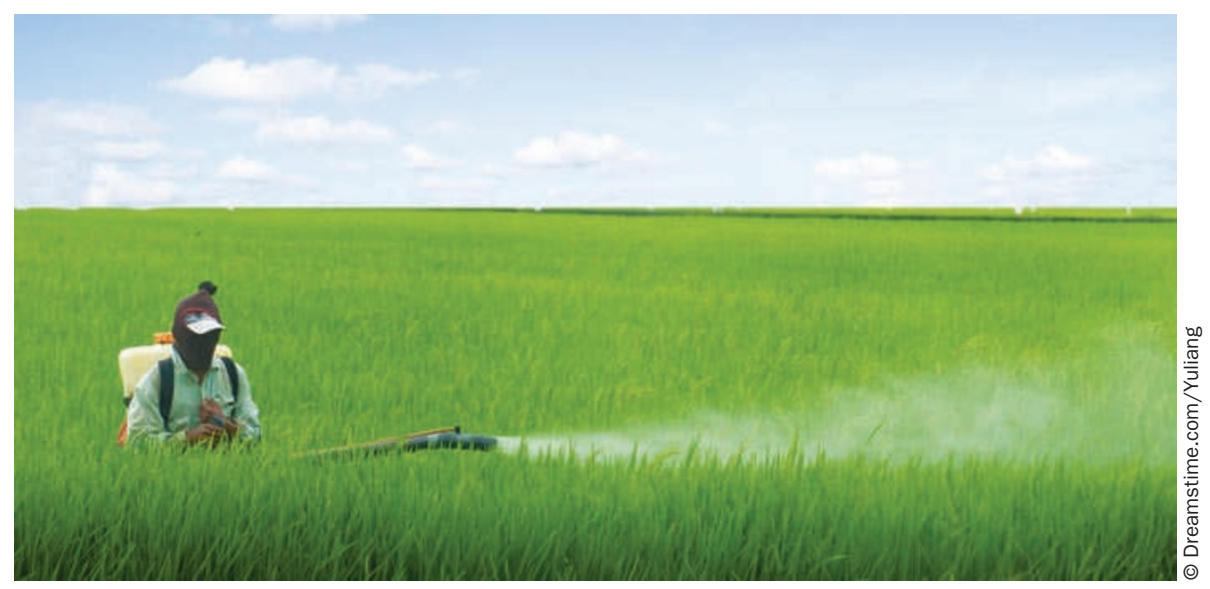

with occupational exposure to pesticides. "When we restricted the outcome to AD, the risk increased," says Hayden.

A number of the most commonly used pesticides such as organophosphates that are known to affect the functioning of the CNS are acetylcholinesterase inhibitors. Organochlorines are also known to affect CNS function and both of these pesticides have been used extensively in the past in agriculture production. The researchers specifically tested whether these two different pesticides were associated with an increased risk of incidental dementia or AD. Analysis of the results revealed that organophosphate exposure was not clearly associated with an increased risk of developing dementia; however, occupational exposure to this pesticide was shown to significantly increase the risk of developing $\mathrm{AD}$ later in life. Organochlorine exposure was not clearly associated with an increased risk of either $\mathrm{AD}$ or dementia.

A substantial proportion of the cohort reported at the start of the study that they had been exposed to both organophosphate and organochlorine pesticides. The investigators performed an additional post hoc analysis to determine whether the risk of developing dementia or $\mathrm{AD}$ was increased in these individuals, but no increased risk was observed in this group. The fact that the association between disease risk and pesticide exposure was moderately stronger when the outcome was restricted to $\mathrm{AD}$ indicates that the deleterious effects of pesticide exposure on cognitve function might be specific to $\mathrm{AD}$.

"Our findings are in line with a large prospective population-based study," explains Hayden. "In that study the authors found an association between pesticide exposure, cognitive performance, and the risk of $\mathrm{AD}$ and Parkinson disease. Moreover, a study of identical twins discordant for $\mathrm{AD}$ has provided additional evidence for this association, and suggests an epigenetic mechanism". The researchers now plan to further investigate the cognitive status of the study participants to determine whether individuals exposed to pesticides have a faster rate of cognitive decline compared with unexposed individuals.

"Further study is needed to determine if there is a causal link between pesticide exposure and increased risk of AD," concludes Hayden.

Nick Jones 